\title{
Integration of major histocompatibility complex, methylation, and transcribed ultra-conserved regions analyses in uremia
}

\author{
Jinjun Qiu', Huiyan $\mathrm{He}^{2}$, Weiguo Sui ${ }^{3}$, Dong'e Tang ${ }^{2}$ and Yong Dai ${ }^{2 *}$ \\ 'Pingshan People's Hospital, Shenzhen, Guangdong, People's Republic of China \\ ${ }^{2}$ Clinical Medical Research Center, The Second Clinical Medical College of Jinan University (Shenzhen People's Hospital), 518020, Shenzhen, Guangdong, \\ People's Republic of China \\ ${ }^{3}$ Nephrology Department of 181st Hospital, Guangxi Key laboratory of Metabolic Diseases Research, 541002, Guilin, Guangxi, People's Republic of China
}

\begin{abstract}
Treatment of uremia is now dominated by dialysis, in some cases, patients are treated with dialysis for decades, but overall outcomes are disappointing. A number of studies have confirmed the relevance of several experimental insights to the pathogenesis of uremia, but the specific biomarkers of uremia have not been fully elucidated. A total of 15 uremia patients and 15 healthy controls were collected in the present study. The aim of this study was to explain the etiology of uremia, MHC gene capture technology, hMeDIP-chip, T-UCR microarray and bioinformatics analysis were utilised in the uremia and normal control group. The result showed 8 CpG methylated enrichment in MHC segment. We found 1 SNP in CpG promoter of lncRNA and 1 SNP in chr6: 28890951-28892013, 1 SNP in CpG chr6:29521110-29521833 and 1 SNP in CpG chr6:30684836-30685503. The CpG methylated corresponding gene wasn't found significant immune correlated process GO term and KEGG pathway enrichment in uremia. In this experiment, T-UCR was not discovered in MHC segment. The T-UCR corresponding gene wasn't found significant immune correlated process GO term and KEGG pathway enrichment too. Analysis of SNP (rs2301754, rs11545587, rs17184255, rs4713354) and expression of the gene in peripheral blood lymphocytes indicated these SNP were associated with the occurrence of uremia. Future studies should examine the roles of these SNP in the pathogenesis of uremia. Integrative analysis technology provided an expansive view of molecular signaling pathways in uremia.
\end{abstract}

\section{Introduction}

Uremia refers to the condition that occurs when kidney function regresses during chronic kidney disease. Chronic kidney disease represents the progressive loss of renal function, and its latest stageuremia, where little or no kidney function is present, requires either transplantation or dialysis [1]. In all stages of the disease, but particularly in uremia, patients present a many-fold increased mortality rate for cardiovascular disease than the general population. Despite intensive research, the pathologic mechanisms of uremia phenotype are still not completely understood and are probably multifactorial. Both genetic and environmental factors have been associated with uremia phenotype, but these factors cannot entirely explain the onset of uremia phenotype. Further studies are still encouraged to shed light on the true associations between uremia and its susceptibility genes. Novel methods should be looked into in this area.

Using a predictive bioinformatics algorithm, Mantila Roosa et al. created a linear model of gene expression and identified 44 transcription factor binding motifs and 29 miRNA binding sites that were predicted to regulate gene expression across the time course. Known and novel transcription factor binding motifs were identified throughout the time course, as were several novel miRNA binding sites. These timedependent regulatory mechanisms may be important in controlling the loading-induced bone formation process [2]. This integrated bioinformatics analysis method may be looked into in our study. The link between $\mathrm{MHC}$ and uremia still unclear. Further investigations are likely to reveal the involvement of MHC in uremia. We are interested in studying MHC, CpG methylated and T-UCR as a first step toward better understanding regulation of gene expression in uremia. We report an expansive view of uremia from an integrated bioinformatics analysis of MHC, CpG methylated and T-UCR data sets.

\section{Materials and methods}

\section{Human subjects}

Thirty subjects were enrolled in the study including 15 uremia patients on dialysis and 15 healthy volunteers. All uremia patients were recruited from the inpatient unit in the Department of Nephrology in the $181^{\text {st }}$ Hospital and were free of active infections, diabetes mellitus, and autoimmune diseases.

Written informed consent was obtained from all the subjects or their guardians. The local Ethics Committee approved the study, and peripheral blood samples were obtained with informed consent from all

Correspondence to: Yong Dai, Clinical Medical Research Center, The Second Clinical Medical College of Jinan University (Shenzhen People's Hospital), 5518020, Shenzhen, Guangdong, People's Republic of China, Fax: +86-75525626750; E-mail: daiyong22@aliyun.com

Key words: major histocompatibility complex, methylation, uremia, transcribed ultraconserved regions

Received: October 22, 2015; Accepted: November 10, 2015; Published: November 16, 2015 
participating individuals. This study abides by the Helsinki Declaration on ethical principles for medical research involving human subjects.

\section{MHC gene capture}

Genomic DNA was isolated from the peripheral blood samples. According to the MHC genomic sequence, a completely complementary probe was designed and fixed on a support, and then applied to the genomic DNA after coupling with a probe connector. Unhybridized probe was washed away; then, probe that had hybridized with the DNA was eluted to directly build a library for DNA sequencing (Hiseq 2000 high-throughput sequencing). MHC region capture technology based on the NimbleGen SeqCap EZ Choice Library that enables deep sequencing coverage of the human MHC region. Data were analyzed by using the chi-squared test with Yates' correction for continuity.

\section{hMeDIP-chip}

Genomic DNA was extracted using a DNeasy Blood \& Tissue Kit (Qiagen, Fremont, CA). One microgram of the sonicated genomic DNA was used for immunoprecipitation using a mouse monoclonal antibody. For DNA labeling, the NimbleGen Dual-Color DNA Labeling Kit was used according to the manufacturer's guidelines that are detailed in the NimbleGen hMeDIP-chip protocol (NimbleGen Systems, Inc., Madison, WI, USA). The microarrays were hybridized in Nimblegen hybridization buffer/hybridization component $\mathrm{A}$ in a hybridization chamber (Hybridization System - Nimblegen Systems, Inc., Madison, WI, USA). For array hybridization, Roche NimbleGen's Promoter plus CpG Island Array was used.

\section{T-UCR microarray analysis}

Sample RNA labeling and array hybridization were performed according to the Agilent One-Color Microarray-Based Gene Expression Analysis protocol (Agilent Technology) with minor modifications. The hybridized arrays were washed, fixed and scanned with using the Agilent DNA Microarray Scanner (part number G2505C). Agilent Feature Extraction software (version 11.0.1.1) was used to analyze acquired array images. Quantile normalization and subsequent data processing were performed with using the GeneSpring GX v12.1 software package (Agilent Technologies).

\section{Bioinformatics analysis}

MHC segment CpG methylated enrichment and difference enrichment analysis: MHC gene capture sequencing segment was chr6:30146860-33375560. To search enrichment location, we analyzed CpG peak in MHC segment.

\section{T-UCR expression in MHC segment}

To search transcript location, we analyzed T-UCR expression in MHC segment.

The effect of CpG methylated level and T-UCR expression level in immunologic process: We analyzed all methylated CpG, T-UCR and their corresponding gene. Then, we analyzed the related gene in immunologic process. In this experiment, to further understand the functions of the gene, we used the online gene ontology tool EASE (http://david.abcc.ncifcrf.gov/ease/ease1.htm. The differential expression gene was to classify in biological process. GO and KEGG pathway mapping of genes were performed by web-accessible DAVID annotation system.

\section{The correlation of MHC mutation and CpG methylated}

To search the correlation, we calculated data of differential CpG methylated, MHC mutation and analyzed correlation coefficient.

\section{Results}

\section{Capturing the quantity of genes and SNP loci in the MHC region}

We obtained 170 genes and 27,454 SNPs by MHC gene capturing and high-throughput sequencing in patients compared with the normal controls.

hMeDIP-chip: The 4063 genes of CpG islands showed significantly different methylation levels in the patients compared with the normal controls.

T-UCR microarray analysis: To identify potential T-UCRs differentially expressed, we performed a fold change filtering in the patients compared with the normal controls. There are 119 potential T-UCRs, which have been collected from authoritative databases such as Refseq, UCSC knowngenes, and Ensembl.

\section{CpG peak in MHC segment}

To search enrichment location, we analyzed CpG peak in MHC segment. The result showed $16 \mathrm{CpG}$ methylated enrichment (Table 1), including 8 in uremia and 8 in the normal controls.

\section{T-UCR expression in MHC segment}

In this study, we analysis all the T-UCR expression, but UCR overlap gene and UCR proximal gene were not discovered in MHC segment.

The effect of CpG methylated level in immunologic process: In this experiment, we annotated $\mathrm{CpG}$ methylated corresponding gene with GO schemes by DAVID gene annotation tool. The genes produced total 55 GO terms in uremia (Table 2), and immune correlated process

Table 1. $16 \mathrm{CpG}$ methylated enrichment in MHC segment.

\begin{tabular}{|l|c|c|c|c|c|}
\hline CpG Name (hg19) & Length (bp) & Control & Uremia & Gene Name & Type \\
\hline chr6:30038881-30039477 & 596 & & 1 & NCRNA00171 & Promoter \\
\hline chr6:30095173-30095610 & 437 & & 1 & TRIM40 & Promoter \\
\hline chr6:32046815-32047094 & 279 & & 1 & TNXB & Intragenic \\
\hline chr6:32163292-32164383 & 1091 & & 1 & PBX2 & Promoter \\
\hline chr6:32163292-32164383 & 1091 & & 1 & GPSM3 & Promoter \\
\hline chr6:32847498-32847846 & 348 & & 1 & PPP1R2P1 & Intragenic \\
\hline chr6:33266302-33267582 & 1280 & & 1 & RGL2 & Promoter \\
\hline chr6:33266302-33267582 & 1280 & & 1 & WDR46 & Promoter \\
\hline chr6:28890951-28892013 & 1062 & 1 & & TRIM27 & Promoter \\
\hline chr6:29521110-29521833 & 723 & 1 & & UBD & Intragenic \\
\hline chr6:30684836-30685503 & 667 & 1 & & MDC1 & Promoter \\
\hline chr6:30684836-30685503 & 667 & 1 & & TUBB & Promoter \\
\hline chr6:31795467-31797384 & 1917 & 1 & & SNORD48 & Promoter \\
\hline chr6:31795467-31797384 & 1917 & 1 & & HSPA1B & Promoter \\
\hline chr6:31795467-31797384 & 1917 & 1 & & C6orf48 & Promoter \\
\hline chr6:31795467-31797384 & 1917 & 1 & & SNORD52 & Promoter \\
\hline
\end{tabular}


Qiu J (2015) Integration of major histocompatibility complex, methylation, and transcribed ultra-conserved regions analyses in uremia

Table 2. The CpG methylated corresponding genes annotation GO terms in uremia.

\begin{tabular}{|c|c|c|c|}
\hline GO Term & Gene Count & P Value & FDR \\
\hline GO:0006350 transcription & 378 & $8.17 \mathrm{E}-12$ & $1.52 \mathrm{E}-08$ \\
\hline $\begin{array}{l}\text { GO:0006355 regulation of transcription, DNA- } \\
\text { dependent }\end{array}$ & 316 & 2.29E-09 & $4.25 \mathrm{E}-06$ \\
\hline GO:0051252 regulation of RNA metabolic process & 321 & $3.44 \mathrm{E}-09$ & $6.39 \mathrm{E}-06$ \\
\hline GO:0045449 regulation of transcription & 436 & 4.54E-09 & $8.43 \mathrm{E}-06$ \\
\hline GO:0007409 axonogenesis & 55 & $3.41 \mathrm{E}-08$ & $6.32 \mathrm{E}-05$ \\
\hline GO:0030182 neuron differentiation & 99 & $5.71 \mathrm{E}-08$ & $1.06 \mathrm{E}-04$ \\
\hline GO:0000904 cell morphogenesis involved in differentiation & 64 & 7.04E-08 & $1.31 \mathrm{E}-04$ \\
\hline GO:0048667 cell morphogenesis involved in neuron differentiation & 57 & $9.74 \mathrm{E}-08$ & $1.81 \mathrm{E}-04$ \\
\hline GO:0006357 regulation of transcription from RNA polymerase II promoter & 145 & $1.85 \mathrm{E}-07$ & $3.44 \mathrm{E}-04$ \\
\hline GO:0048812 neuron projection morphogenesis & 56 & $4.70 \mathrm{E}-07$ & $8.72 \mathrm{E}-04$ \\
\hline GO:0048666 neuron development & 77 & $1.62 \mathrm{E}-06$ & 0.003011 \\
\hline GO:0006355 regulation of transcription, DNA-dependent & 61 & 4.61E-06 & 0.008556 \\
\hline GO:0051252 regulation of RNA metabolic process & 60 & $9.58 \mathrm{E}-06$ & 0.017786 \\
\hline GO:0045449 regulation of transcription & 58 & $1.00 \mathrm{E}-05$ & 0.018585 \\
\hline GO:0007409 axonogenesis & 61 & $1.83 \mathrm{E}-05$ & 0.034300 \\
\hline GO:0030182 neuron differentiation & 76 & $2.01 \mathrm{E}-05$ & 0.037274 \\
\hline GO:0000904 cell morphogenesis involved in differentiation & 36 & $2.52 \mathrm{E}-05$ & 0.046809 \\
\hline GO:0048667 cell morphogenesis involved in neuron differentiation & 39 & $5.75 \mathrm{E}-05$ & 0.106653 \\
\hline GO:0006357 regulation of transcription from RNA polymerase II promoter & 30 & $9.17 \mathrm{E}-05$ & 0.170063 \\
\hline GO:0048812 neuron projection morphogenesis & 80 & $9.86 \mathrm{E}-05$ & 0.182883 \\
\hline GO:0048666 neuron development & 75 & $1.12 \mathrm{E}-04$ & 0.208311 \\
\hline GO:0031175 neuron projection development & 46 & $1.31 \mathrm{E}-04$ & 0.242746 \\
\hline GO:0032990 cell part morphogenesis & 68 & $1.90 \mathrm{E}-04$ & 0.351983 \\
\hline GO:0048858 cell projection morphogenesis & 114 & $2.21 \mathrm{E}-04$ & 0.409970 \\
\hline GO:0007389 pattern specification process & 68 & $2.49 \mathrm{E}-04$ & 0.461054 \\
\hline GO:0000902 cell morphogenesis & 106 & $2.85 \mathrm{E}-04$ & 0.528252 \\
\hline GO:0007156 homophilic cell adhesion & 71 & $3.38 \mathrm{E}-04$ & 0.625256 \\
\hline GO:0030900 forebrain development & 116 & $3.41 \mathrm{E}-04$ & 0.630759 \\
\hline GO:0006355 regulation of transcription, DNA-dependent & 105 & $3.58 \mathrm{E}-04$ & 0.662752 \\
\hline GO:0051252 regulation of RNA metabolic process & 103 & $3.80 \mathrm{E}-04$ & 0.703840 \\
\hline GO:0045449 regulation of transcription & 106 & $3.93 \mathrm{E}-04$ & 0.727092 \\
\hline GO:0007409 axonogenesis & 87 & 4.04E-04 & 0.746483 \\
\hline GO:0030182 neuron differentiation & 73 & $4.18 \mathrm{E}-04$ & 0.772374 \\
\hline GO:0000904 cell morphogenesis involved in differentiation & 103 & 4.68E-04 & 0.864433 \\
\hline GO:0048667 cell morphogenesis involved in neuron differentiation & 60 & 4.69E-04 & 0.866098 \\
\hline GO:0006357 regulation of transcription from RNA polymerase II promoter & 71 & $5.54 \mathrm{E}-04$ & 1.022866 \\
\hline GO:0048812 neuron projection morphogenesis & 93 & $6.35 \mathrm{E}-04$ & 1.171092 \\
\hline GO:0048666 neuron development & 60 & $6.70 \mathrm{E}-04$ & 1.236425 \\
\hline GO:0031175 neuron projection development & 94 & 7.01E-04 & 1.293726 \\
\hline GO:0032990 cell part morphogenesis & 95 & 7.33E-04 & 1.351237 \\
\hline GO:0048858 cell projection morphogenesis & 99 & $8.20 \mathrm{E}-04$ & 1.510345 \\
\hline GO:0007389 pattern specification process & 115 & $9.05 \mathrm{E}-04$ & 1.666747 \\
\hline GO:0000902 cell morphogenesis & 56 & 0.001007 & 1.853066 \\
\hline GO:0007156 homophilic cell adhesion & 33 & 0.001143 & 2.099637 \\
\hline GO:0030900 forebrain development & 87 & 0.001209 & 2.220594 \\
\hline GO:0007411 axon guidance & 126 & 0.001287 & 2.362594 \\
\hline GO:0032989 cellular component morphogenesis & 87 & 0.001377 & 2.525408 \\
\hline GO:0030030 cell projection organization & 118 & 0.001636 & 2.992538 \\
\hline GO:0003002 regionalization & 60 & 0.001677 & 3.066779 \\
\hline GO:0043009 chordate embryonic development & 87 & 0.001789 & 3.269362 \\
\hline $\begin{array}{l}\text { GO:0045935 positive regulation of nucleobase, nucleoside, nucleotide and nucleic acid } \\
\text { metabolic process }\end{array}$ & 30 & 0.001931 & 3.523561 \\
\hline GO:0009792 embryonic development ending in birth or egg hatching & 53 & 0.002101 & 3.828081 \\
\hline GO:0016192 vesicle-mediated transport & 12 & 0.002437 & 4.427231 \\
\hline GO:0045892 negative regulation of transcription, DNA-dependent & 12 & 0.002437 & 4.427231 \\
\hline GO:0051173 positive regulation of nitrogen compound metabolic process & 118 & 0.002722 & 4.933267 \\
\hline
\end{tabular}

Note: If p-value $<0.005$, it is considered significant. The False Discovery Rate (FDR) of a set of predictions is the expected percent of false predictions in the set of predictions. Meanwhile an FDR $<5 \%$ might be quite meaningful. 
GO term such as GO:0006955-immune response wasn't found significant enrichment.

In addition, we obtained 4 KEGG pathways of the genes in uremia (Table 3), immune correlated process KEGG pathway wasn't significantly enriched.

The effect of T-UCR expression level in immunologic process: In this experiment, we annotated T-UCR corresponding gene with GO schemes by DAVID gene annotation tool. The genes produced total 52 GO terms in uremia (Table 4), and immune correlated process GO term wasn't found significant enrichment. In addition, we don't obtained immune correlated process KEGG pathway of the genes in SLE. They weren't significantly enriched.

The correlation of MHC mutation and CpG methylated: In this experiment, we found 1 SNP in CpG promoter of lncRNA and 1 SNP in chr6: 28890951-28892013,1 SNP in CpG chr6:29521110-29521833 and 1 SNP in CpG chr6:30684836-30685503 (Table 5).

\section{Discussion}

The first genetic factors to be identified as important in the pathogenesis of uremia were those of the MHC on chromosome 6. It is now widely accepted that MHC genes constitute a part of the genetic susceptibility to uremia. Previous studies in uremia have lacked statistical power and genetic resolution to fully define MHC influences. In this research, we tried to identify MHC, CpG methylated and T-UCR, and reveal potential mechanism in uremia by a novel and combinatorial approach involving MHC gene capture technology, hMeDIP-chip, T-UCR microarray, and bioinformatic analysis. 27,454 SNPs were detected significantly different which may be involved in uremia. Moreover, in this study, we integrated the datasets and identified 4 most important SNPs in uremia. Function research on these SNPs is in our plan.

It is reported that $\mathrm{H} 3 \mathrm{~K} 4 \mathrm{me} 3$ altered in uremia patients but not in healthy people [3]. Their results indicate that H3K9 trimethylation is involved in unphysiological uremic environment and these novel candidate genes may become potential biomarkers or future therapeutic targets [4]. Epigenetic events play a central role in the priming, differentiation and subset determination of $\mathrm{T}$ lymphocytes. CpG-DNA methylation and post-translational modifications to histone tails are the two most well accepted epigenetic mechanisms. The involvement of epigenetic mechanisms in the pathogenesis of uremia has been suggested A better understanding of the molecular events that contribute to epigenetic alterations and subsequent immune imbalance is essential for the establishment of disease biomarkers and identification of potential therapeutic targets. These findings may facilitate the selection of better target molecules for further studies. Our study might also aid in suggesting new pathways to be studied in a more focused approach with confirmation at the protein levels and investigation of the clinical significance.

Long non-coding RNAs (lncRNAs) are transcripts longer than $\sim 200$ nucleotides with little or no protein-coding capacity [5]. Growing evidence shows that lncRNAs present important function in development and are associated with many human diseases such as cancers, Alzheimer disease, and heart diseases. T-UCR transcripts are a novel class of lncRNAs transcribed from ultraconserved regions (UCRs). UCRs are a class of 481 noncoding sequences located in both intra- and inter-genic regions of the genome. UCRs are absolutely conserved (100\%) between the orthologous regions of the human, rat, and mouse genomes, and are actively transcribed. It has recently been proven in cancer systems that differentially expressed T-UCRs could alter the functional characteristics of malignant cells. Recent data suggest that T-UCRs are altered at the transcriptional level in human tumorigenesis and the aberrant T-UCRs expression profiles can be used to differentiate human cancer types [6,7]. Researchers observed that DNA hypomethylation induces release of T-UCR silencing in cancer cells. The analysis of a large set of primary human tumors demonstrated that hypermethylation of the described T-UCR CpG islands was a common event among the various tumor types [8]. However, in our study, we integrated the MHC and T-UCR datasets. We examined the expression levels of T-UCR in MHC segment by T-UCR microarray. We annotated T-UCR corresponding gene by DAVID gene annotation tool. The immune correlated process GO term and KEGG pathway wasn't found significant enrichment. T-UCR expression levels were not correlated with commonly used clinicopathological features of uremia.

\section{Conclusions}

Taken together, we identified 4 most important SNPs (rs2301754, rs11545587, rs17184255, rs4713354) in uremia. Our work indicates that SNPs in MHC segment are potential biomarkers and probable factors involved in the pathogenesis of uremia. However, further studies are required to investigate the mechanism by which polymorphisms in this gene lead to uremia. A major advantage of combining multiple planes of measurement is the ability to dissect mechanisms not apparent in a single dimension. Integrating MHC, CpG methylated and T-UCR data sets is a powerful strategy for understanding uremia biology. Our findings proved insights into the anomalous regulated SNPs' potential contribution to the abnormalities in uremia and could help us to structure antenatal diagnostic biomarkers of uremia, as well as get the novel therapeutic targets in the treatment of individual with uremia. Besides, our study of SNPs may lead to finding novel methods to treat and prevent other diseases.

\section{Acknowledgements}

The authors thank the patients with uremia and healthy volunteers

Table 3. The CpG methylated corresponding genes annotation KEGG pathways in uremia.

\begin{tabular}{|l|c|c|c|}
\hline Pathways & Gene Count & P Value & FDR \\
\hline hsa04012:ErbB signaling pathway & 24 & $3.41 \mathrm{E}-04$ & 0.418186 \\
\hline hsa05220:Chronic myeloid leukemia & 20 & 0.001899 \\
\hline hsa04144:Endocytosis & 38 & 0.002299 & 2.308109 \\
\hline hsa05214:Glioma & 17 & 0.004113 & 2.787276 \\
\hline
\end{tabular}

Note: If p-value $<0.005$, it is considered significant. The False Discovery Rate (FDR) of a set of predictions is the expected percent of false predictions in the set of predictions. Meanwhile an FDR $<5 \%$ might be quite meaningful. 
Table 4. The T-UCR corresponding genes annotation GO terms in uremia

\section{GO Term}

GO:0045449 regulation of transcription

GO:0051252 regulation of RNA metabolic process

GO:0006350 transcription

GO:0006357 regulation of transcription from RNA polymerase II promoter

GO:0006355 regulation of transcription, DNA-dependent

GO:0003002 regionalization

GO:0045941 positive regulation of transcription

GO:0045935 positive regulation of nucleobase, nucleoside, nucleotide and nucleic acid

metabolic process

GO:0010628 positive regulation of gene expression

GO:0051173 positive regulation of nitrogen compound metabolic process

GO:0016071 mRNA metabolic process

GO:0048598 embryonic morphogenesis

GO:0051254 positive regulation of RNA metabolic process

GO:0006397 mRNA processing

GO:0007389 pattern specification process

GO:0006396 RNA processing

GO:0045944 positive regulation of transcription from RNA polymerase II promoter

GO:0045893 positive regulation of transcription, DNA-dependent

GO:0048706 embryonic skeletal system development

GO:0008380 RNA splicing

GO:0009952 anterior/posterior pattern formation

GO:0031328 positive regulation of cellular biosynthetic process

GO:0048562 embryonic organ morphogenesis

GO:0010557 positive regulation of macromolecule biosynthetic process

GO:0009891 positive regulation of biosynthetic process

GO:0031327 negative regulation of cellular biosynthetic process

GO:0010604 positive regulation of macromolecule metabolic process

GO:0048568 embryonic organ development

GO:0030900 forebrain development

GO:0010558 negative regulation of macromolecule biosynthetic process

GO:0009890 negative regulation of biosynthetic process

GO:0010605 negative regulation of macromolecule metabolic process

GO:0001501 skeletal system development

GO:0010629 negative regulation of gene expression

GO:0021537 telencephalon development

GO:0051172 negative regulation of nitrogen compound metabolic process

GO:0021543 pallium development

GO:0016481 negative regulation of transcription

GO:0045934 negative regulation of nucleobase, nucleoside, nucleotide and nucleic acid

metabolic process

GO:0051253 negative regulation of RNA metabolic process

GO:0048704 embryonic skeletal system morphogenesis

GO:0045892 negative regulation of transcription, DNA-dependent

GO:0030902 hindbrain development

GO:0048705 skeletal system morphogenesis

GO:0000122 negative regulation of transcription from RNA polymerase II promoter

GO:0007411 axon guidance

GO:0030326 embryonic limb morphogenesis

GO:0035113 embryonic appendage morphogenesis

GO:0009954 proximal/distal pattern formation

GO:0009953 dorsal/ventral pattern formation

GO:0051051 negative regulation of transport

GO:0045665 negative regulation of neuron differentiation

\begin{tabular}{|c|c|}
\hline Gene Count & \\
\hline 240 & \\
\hline 176 & \\
\hline 191 & \\
\hline 87 & \\
\hline 167 & \\
\hline 35 & \\
\hline 66 & \\
\hline 70 & \\
\hline 66 & \\
\hline
\end{tabular}

P Value

$8.45 \mathrm{E}-14$

$1.11 \mathrm{E}-11$

$3.49 \mathrm{E}-10$

4.20E-10

$5.03 \mathrm{E}-10$

$1.59 \mathrm{E}-08$

$1.74 \mathrm{E}-07$

$3.44 \mathrm{E}-07$

$5.15 \mathrm{E}-07$

5.44E-07

$6.14 \mathrm{E}-07$

8.78E-07

9.23E-07

$1.12 \mathrm{E}-06$

$1.22 \mathrm{E}-06$

$1.30 \mathrm{E}-06$

$1.60 \mathrm{E}-06$

$1.60 \mathrm{E}-06$

2.01E-06

$2.10 \mathrm{E}-06$

2.31E-06

2.61E-06

$3.21 \mathrm{E}-06$

3.84E-06

4.37E-06

6.09E-06

9.65E-06

9.77E-06

$1.01 \mathrm{E}-05$

$1.13 \mathrm{E}-05$

$1.17 \mathrm{E}-05$

2.79E-05

$3.18 \mathrm{E}-05$

6.67E-05

9.53E-05

2.63E-04

2.97E-04

3.37E-04

3.48E-04

4.47E-04

4.50E-04

6.39E-04

7.10E-04

$9.18 \mathrm{E}-04$

0.001139

0.001591

0.001782

0.001782

0.001812

0.002589

0.002629

0.002778
FDR

$1.52 \mathrm{E}-10$

$1.99 \mathrm{E}-08$

$6.27 \mathrm{E}-07$

7.54E-07

9.05E-07

2.85E-05

3.13E-04

6.17E-04

9. $25 \mathrm{E}-04$

9.78E-04

0.001104

0.001579

0.001659

0.002013

0.002200

0.002335

0.002875

0.002876

0.003616

0.003773

0.004161

0.004690

0.005772

0.006907

0.007853

0.010950

0.017339

0.017567

0.018085

0.020368

0.021059

0.050223

0.057209

0.119750

0.171182

0.470982

0.531764

0.603146

0.623182

0.800670

1.142592

1.268796

1.636420

2.027251

2.820892

3.154926

3.154926

3.206449

4.553220

4.620857

4.877394

4.825061

Note: If p-value $<0.005$, it is considered significant. The False Discovery Rate (FDR) of a set of predictions is the expected percent of false predictions in the set of predictions. Meanwhile an FDR $<5 \%$ might be quite meaningful. 
Table 5. 4 CpG methylated SNPs of MHC segment in uremia.

\begin{tabular}{|c|c|c|c|}
\hline Chromosome segment & SNP & Gene & Function \\
\hline chr6: 30039098 & rs2301754 & RNF3 & exonic \\
\hline chr6: 28891522 & rs11545587 & TRIM27 & UTR5 \\
\hline chr6: 29521289 & rs17184255 & LOC100507362 & intergenic \\
\hline chr6: 30685420 & Rs4713354 & MDC1 & UTR5 \\
\hline
\end{tabular}

who participated in this study. This work was supported by grants from the Health system research project of Pingshan, Shenzhen (no. 201417) and Incubation fund of medical and health development of Pingshan (no.201317) Bioinformatics analysis was performed by Shanghai BIOTREE biotech Co. Ltd., Shanghai, China.

\section{Disclosure}

The authors of this manuscript have no conflicts of interest to disclose.

\section{References}

1. Ingrosso D, Perna AF (2009) Epigenetics in hyperhomocysteinemic states. A special focus on uremia. Biochimica et Biophysica Acta 1790: 892-899. [Crossref]

2. Mantila Roosa SM, Turner CH, Liu Y (2012) Regulatory mechanisms in bone following mechanical loading. Gene Regul Syst Biol 6: 43-53. [Crossref]

3. Zhang L, Dai Y, Wang L, Peng W, Zhang Y, et al. (2011) CpG Array Analysis of Histone H3 Lysine 4 Trimethylation in Peripheral Blood Mononuclear Cells of Uremia Patients. DNA and Cell Biology 30: 179-186. [Crossref]

4. Sui W, He H, Yan Q, Chen J, Zhang R, et al. (2013) Genome-wide analysis of histone $\mathrm{H} 3$ lysine 9 trimethylation by ChIP-seq in peripheral blood mononuclear cells of uremia patients. Hemodial Int 17: 493-501. [Crossref]

5. Yan B, Tao ZF, Li XM, Zhang H, Yao J, et al. (2014) Aberrant expression of long noncoding RNAs in early diabetic retinopathy. Invest Ophthalmol Vis Sci 55: 941-51. [Crossref]

6. Peng JC, Shen J, Ran ZH (2013) Transcribed ultraconserved region in human cancers RNA Biol 10(12): 1771-7. [Crossref]

7. Sana J, Hankeova S, Svoboda M, Kiss I, Vyzula R, et al. (2012) Expression levels of transcribed ultraconserved regions uc. 73 and uc.388 are altered in colorectal cancer. Oncology 82: 114-8. [Crossref]

8. Lujambio A, Portela A, Liz J, Melo SA, Rossi S, et al. (2010) CpG island hypermethylation-associated silencing of non-coding RNAs transcribed from ultraconserved regions in human cancer. Oncogene 29: 6390-401. [Crossref]

Copyright: (C2015 Qiu J. This is an open-access article distributed under the terms of the Creative Commons Attribution License, which permits unrestricted use, distribution, and reproduction in any medium, provided the original author and source are credited. 